\title{
A WIRELESS EEG ACQUISITION SYSTEM WITH THERMOELECTRIC SCAVENGING MICRODEVICE
}

\author{
J. P. Carmo, L. M. Goncalves, R. P. Rocha and J. H. Correia \\ University of Minho, Dept. Industrial Electronics, Campus Azurem, 4800-058 Guimaraes, Portugal \\ jcarmo@dei.uminho.pt
}

Keywords: Wireless EEG, $\mathrm{IrO}_{2}$ electrodes, Thermoelectric microsystems, Energy scavenging.

\begin{abstract}
This paper presents a wireless EEG acquisition system powered by a thermoelectric energy scavenger, which was optimised to convert the small thermal power available in the human-body. The wireless EEG system is composed with up to four EEG electrodes. A radio-frequency (RF) transceiver for operation in the $2.4 \mathrm{GHz}$ ISM band, was optimised and fabricated in the UMC RF $0.18 \mu \mathrm{m}$ CMOS process. The receiver has a sensitivity of $-60 \mathrm{dBm}$ and consumes $6.3 \mathrm{~mW}$ from a $1.8 \mathrm{~V}$ supply. The transmitter delivers an output power of $0 \mathrm{dBm}$ with a power consumption of $11.2 \mathrm{~mW}$. Because listening and emitting are power-intensive activities, innovative topics concerning efficient power management was taken into account during the design of the RF CMOS transceiver.
\end{abstract}

\section{INTRODUCTION}

Energy scavengers are currently emerging for a number of applications from automotive to medicine. Micro energy scavengers are small electromechanical devices which harvest ambient energy and convert it into electricity. Energy scavengers could harvest different types of energies. Solar energy can be harvested with photovoltaic solar cells, thermal energy can be harvested with thermoelectric generators, mechanical energy can be harvested with piezoelectric, electromagnetic or electrostatic converters, and finally electromagnetic energy can be harvested through RF resonators. It exists two types of energy scavenging systems: macro energy scavengers, typically in the $\mathrm{cm}^{3}$ range, and micro energy scavengers, typically in the $\mathrm{mm}^{3}$ range and manufactured using micromachining techniques. Micro energy scavengers are still in the R\&D phase. Direct thermal-to-electric energy conversion without moving mechanical parts is attractive for a wide range of applications because it provides compact and distributed power, quiet operation, and is usually environmentally friendly. Thus, worldwide efforts are undertaken to expand the technology of thermoelectric devices into the field of micro-systems technologies (MEMS). Previsions made by the specialists of the microsystems area, shows that the most expected growth of these devices, will be with medical applications.

An emerging technology for ultra-low power communication platforms triggered renewed interest in power sources for wireless-sensor, in special wireless-wearable-sensors, with power consumption nodes of few $\mathrm{mW}$. Today, almost all of these platforms are designed to run on batteries which not only have a very limited lifetime, but are also in many areas a cost-prohibitive solution. An attractive alternative is powering the sensors with energy harvested from the environment. The developed project aims at a solution for energy microgeneration through energy harvesting by taking advantage of temperature differences. A viable energy source for low-powered devices such as micro sensor systems, ZigBee chipsets, wearable electronics, implantable medical devices, active RFID tags and many other applications is proposed, provided a temperature difference exists, between the two surfaces of a thermoelectric microgenerator (in a wearable device, the difference between the body and environment can be tens of degree, depending on the environment temperature). This temperature difference can be converted into electrical energy using the Seebeck principle. Since many of wireless sensors are powered in a peak basis (e.g., the transmission of data needs much more current than standby or receiving mode) and the temperature gradient could not always be present, the energy is stored in a 
rechargeable thin-film battery of the Li-ion type (integrated in the system). Ultra-low power electronics performs DC-DC rectification with a variable conversion factor and recharge the battery on optimal conditions. Since a small volume is required, integration into an IC is desirable. A single-chip regulated thermoelectric power source is the final goal to be achieved.

\section{THERMOELECTRIC GENERATOR}

Future investigations, must prove if the operation from low temperature gradients (a minimum temperature difference of $3{ }^{\circ} \mathrm{C}$ between ambient and target thermo-source must provide an IC-compatible voltage) and the power of $2 \mathrm{~mW} / \mathrm{cm}^{2}$ are possible to be obtained. The fabrication of macro-scale thermoelectric devices is based on standard technologies for decades. Bismuth and antimony tellurides was be used as thermoelectric materials since these materials have the highest performance figure-of-merit (ZT) at room temperature (Gonçalves et al, 2006). The co-deposition method was used to fabricate these thermoelectric thin films. A very stable evaporation rate of each element $(\mathrm{Bi} / \mathrm{Te}$ and $\mathrm{Sb} / \mathrm{Te}$ for the bismuth telluride and antimony telluride, respectively) allows the deposition of polycrystalline n-type and p-type materials, when the substrate is heated in the range 200-300 ${ }^{\circ} \mathrm{C}$. The design of a thermoelectric microdevice, with vertical microcolumns, connected in series by metal contact areas, requires the application of microsystem technologies (Gonçalves et al, 2007). Reactive ion etching, lift-of and wet-etching (hydrochloridric acid / nitric acid solution) techniques were tested to create the vertical columns. A thin $(50 \mathrm{~nm})$ layer of nickel (deposited by e-beam) interfaces between the thermoelectric material and the metal contact areas $(1 \mu \mathrm{m}$ of aluminum), preventing the diffusion of the metal from the contacts into the thermoelectric film. The contact resistance plays a major role in the performance of the device, and a value smaller then $1 \times 10^{-6} \mathrm{ohm} . \mathrm{cm}^{2}$ was achieved. A silicon substrate was used for integration with microelectronics, while at same time providing good thermal contact with heat source and sink. The fabricated battery, as well as, all the electronic circuitry to receive the energy and to recharge the thin-film integrated Li-ion battery (open circuit voltages between $1.5 \mathrm{~V}$ and $4.2 \mathrm{~V}$, maximum current of few $\mathrm{mA} / \mathrm{cm}^{2}$ with a charge-storage capacity around $100 \mu \mathrm{Ah} / \mathrm{cm}^{2}$ ), was placed on the bottom side of the generator. Thin-film solid-state batteries show a very high life cycle and are intrinsically safe (Bates at al, 2000).

The Figure 1 shows an artist impression of a thermoelectric microdevice, with vertical microcolumns, connected in series by metal contact areas. On this thermoelectric generator will be placed the thin-film integrated battery and all the electronic circuitry to receive the energy and to recharge the battery. The application of this thermoelectric scavenging energy systems, is in the powering of a wireless EEG acquisition system.

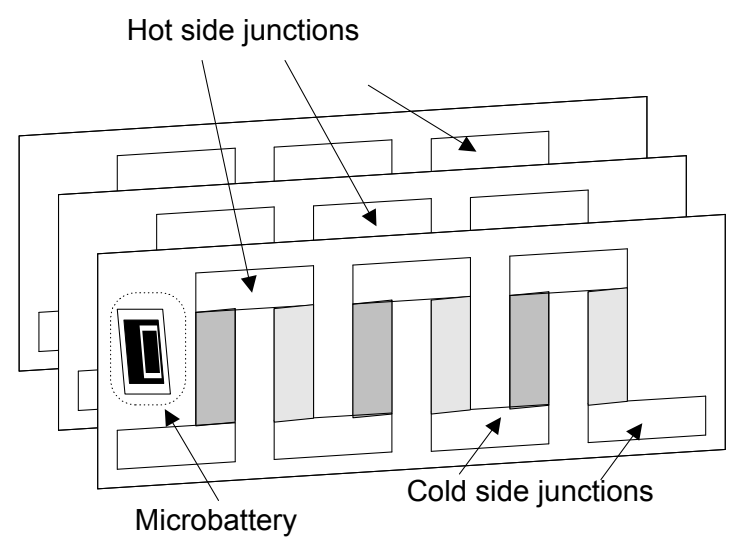

Figure 1: An artist impression of a thermoelectric microdevice.

\section{EEG ACQUISITION SYSTEM}

The standard wireless EEG solutions use a braincap with wires running from the electrodes position to a bulky central unity (amplification, signal filtering and analog-to-digital conversion) (IMEC 2003). A more interesting solution is to use compact wireless EEG modules, where the electronics, the antenna and each electrode are mounted together. The power supply for these modules is obtained locally from the thermoelectric generator. Also, it is possible to integrate additional electronics (amplification, filtering and high-resolution digital conversion), for local signal processing in these small-size individual wireless EEG modules.

Bipolar or unipolar electrodes can be used in the EEG measurement. In the first method the potential difference between a pair of electrodes is measured. In the second method, the potential of each electrode is compared, either to a neutral electrode or to the average of all electrodes. Figure 2 shows the full block diagram of the wireless EEG module, where it 
can be seen the electrode connected to an amplifier, followed by an analog-to-digital converter (ADC). In order to meet the EEG specifications, the amplifier was designed to have enough gain, to amplify signals with amplitudes of only $70 \mu \mathrm{V}$. The $\mathrm{ADC}$ must have at least a resolution of 22 bits and a minimum sampling frequency of $2000 \mathrm{~Hz}$.

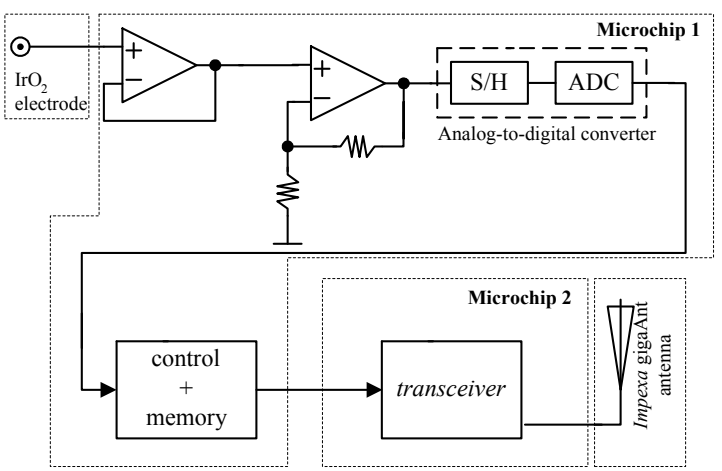

Figure 2: Wireless EEG module. Note that the neutral electrode, which is connected to the grounds of the module it is not shown.

This wireless acquisition system uses a RF link to communicate with an external base-station. It was used the UMC RF $0.18 \mu \mathrm{m}$ CMOS process in the design and fabrication of the RF transceiver. Figure 3 shows the block schematic of this RF transceiver, which consists of a receiver, a transmitter, an antenna-switch and a Phase-locked Loop (PLL) as a frequency syntheziser. The RF transceiver was built to operate at the $2.4 \mathrm{GHz}$ ISM band. The receiver adopts a direct demodulation, by means of envelope detection and has a sensibility of $-60 \mathrm{dBm}$ for power consumption of $6.3 \mathrm{~mW}$ from a $1.8 \mathrm{~V}$ supply. The transmitter delivers an output power of $0 \mathrm{dBm}$ with a power consumption of $11.2 \mathrm{~mW}$. These characteristics fullfill the requirements for communications up to ten meters, with a bit error probability less than $10^{-6}$.

Moreover, without proper design, communication tasks may increase network power consumption significantly because listening and emitting are power-intensive activities (Enz et al, 2004). Thus, in order to optimise the power consumption, the RF transceiver design predicted the use of control signals. With these control signals it is possible to enable and disable all the transceiver subsystems. These signals allows, e.g., to switch off the receiver when a RF signal is being transmitted, to switch off the transmitter when a RF signal is being received, and allows the transceiver to enter to sleep when RF signals are neither being transmitted, nor being received. The Figure 4 shows a die photograph of the RF transceiver.

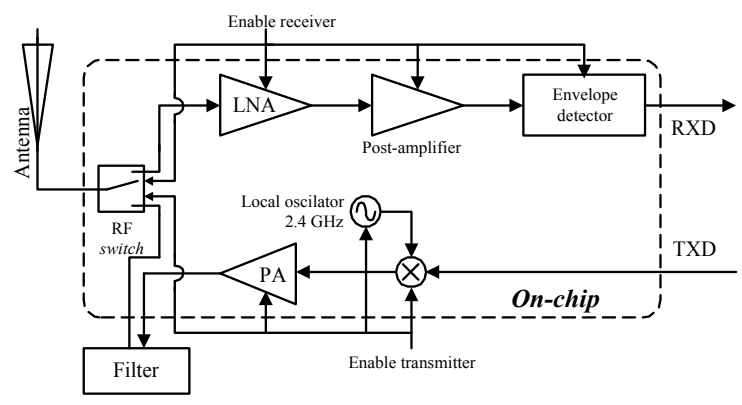

Figure 3: The block schematic of the transceiver.

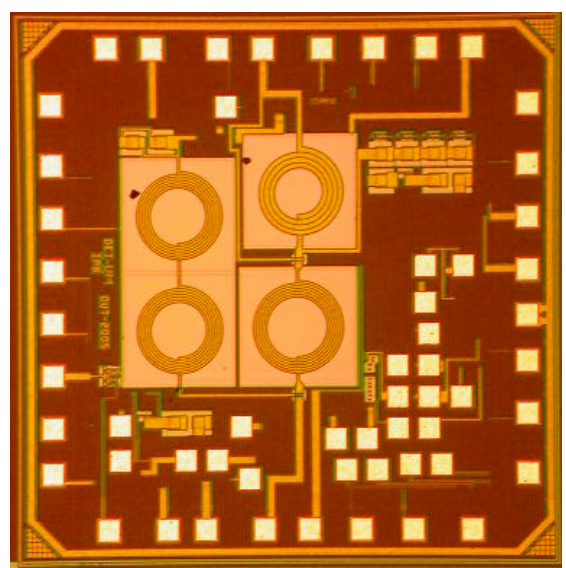

Figure 4: A die photograph of the RF transceiver.

The electrodes to acquire the EEG signals, with the proposed wireless acquisition system, are of sputtered iridium oxide $\left(\mathrm{IrO}_{2}\right)$ type. The experimental results shown a better performance of the sputtered $\mathrm{IrO}_{2}$ electrodes compared with the standard sintered $\mathrm{Ag} / \mathrm{AgCl}$ ring electrodes (Slavcheva et al., 2004). These results promise a new opportunity for the application of a dry $\mathrm{IrO}_{2}$ electrodes in our wireless EEG modules, without the need to use conductive gel in the interface between the electrode and the skin. This will allow patients to wear a brain cap with the electrodes and maintain their mobility, while simultaneously having their electrical brain activity monitored. The Figure 5 shows an artist impression of the thermoelectric scavenging system and an wireless EEG module, both attached to a cap (the zoomed part in that Figure). The temperature gradient between forehead and the environment will generate energy in the thermoelectric microdevice and charge the solidstate thin-film battery. 
The modules must offer the plug-and-play feature, in order to mount distributed networks in the patient's head. Moreover, as the EEG data is periodically acquired in all the modules, thus the latencies of data transmissions are not allowed. The proposed EEG modules uses a communication protocol that overcomes these problems (Afonso et al, 2006). This protocol combines the distributed and coordination modes, e.g., when a new module is putted in the head of patient, a contention based time interval is used to make the registration request in the network. A contention-less time interval, constituted by time-slots, is granted to the new EEG module if the registration is successful completed on the network. The maximum number of simultaneous modules is limited to the number of time-slots in the contentionfree interval.

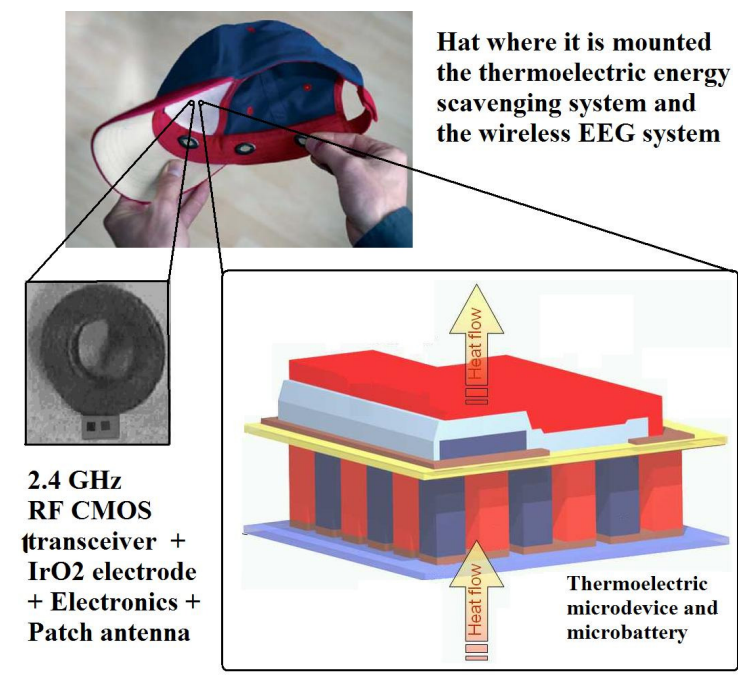

Figure 5: An artist impression of the thermoelectric scavenging system and an wireless EEG module.

\section{CONCLUSIONS}

This paper presented a thermoelectric scavenging energy system, whose final goal is to supply a wireless EEG system. The wireless EEG system is composed by plug-and-play modules, one for each electrode. Each wireless module is composed by an electrode, processing electronics, a radio-frequency transceiver and an associated antenna.

The RF transceiver was fabricated in the UMC RF $0.18 \mu \mathrm{m}$ CMOS process, for the operation in the $2.4 \mathrm{GHz}$ ISM band, in order to optimise the consumed power.
The use of microsystems techniques, makes possible the integration of the whole thermoelectric scavenging system, in an wireless EEG system.

\section{ACKNOWLEDGEMENTS}

The authors would like to thanks the Portuguese Foundation for Science and Technology (FCT/PTDC/EEA-ENE/66855/2006 project).

\section{REFERENCES}

Afonso, J., et al, 2006, MAC protocol for low-power realtime wireless sensing and actuation, in Proc. of the 13th IEEE International Conference on Electronics, Circuits and Systems, Nice, France, pp. 1248-1251.

Bates, J., et al, 2000, Thin-film lithium and lithium-ion batteries, Solid State Ionics, Vol. 135, pp. 33-45.

Enz, C., et al, 2004, WiseNET: An ultralow-power wireless sensor network solution", IEEE Computer, Vol. 37, pp. 62-70.

Gonçalves, L. M., et al, 2006, Thermoelectric properties of $\mathrm{Bi} 2 \mathrm{Te} 3 / \mathrm{Sb} 2 \mathrm{Te} 3$ thin films, Materials Science Forum, Vols. 514-516, pp. 156-160.

Gonçalves, L. M., et al, 2007, Fabrication of flexible thermoelectric microcoolers using planar thin-film thecnologies, Journal of Micromechanics and Microengineering, Vol. 17, pp. 168-173.

IMEC, 2003, IMEC press releases, Ambulatory EEG, Human ++ EU project, pp. 1-2.

Slavcheva, E., et al, 2004, Sputtered iridium oxide films as charge injection material for functional electrostimulation, Journal of the Electrochemical Society, Vol. 151, Nº , pp. 226-237. 